\title{
Non-Muscle Invasive Bladder Cancer: A Review of the Current Trend in Africa
}

\author{
Ayun Cassella, d, Bashir Yunusa ${ }^{\mathrm{b}}$, Mohamed Jalloha ${ }^{\mathrm{a}}$, Mouhamadou M. Mbodji ${ }^{\mathrm{a}}$, \\ Abdourahmane Diallo ${ }^{a}$, Madina Ndoye ${ }^{a}$, Yoro Diallo ${ }^{c}$, Issa Labou ${ }^{a}$, \\ Lamine Niang ${ }^{\mathrm{a}}$, Serigne M. Gueye ${ }^{\mathrm{a}}$
}

\begin{abstract}
Bladder cancer is the fourth most common cancer in men and the 11th most common cancer in woman accounting for $6.6 \%$ of all cancer cases. Approximately $70-75 \%$ bladder cancers are non-muscle invasive bladder cancer (NMIBC). A few African studies have provided considerable rates of NMIBC as compared to western settings $70 \%$ to $85 \%$. Critical step in the management of NMIBC is to prevent tumor recurrence which include transurethral resection of the bladder tumor (TURBT) for staging and histological diagnosis. A second TURBT for high grade tumor, T1 tumors and intravesical adjuvant chemotherapy and immunotherapy are essential to reduce recurrence rate. Nevertheless, variant histology, multiple, progressive and recurrent high-grade tumors are best treated with early radical cystectomy. The African literature is scanty on the management of NMIBC. Most of the histological types are squamous cell bladder cancer and may not conform to transurethral resection only but rather radical cystectomy. Most of these patients are not suitable for any form of treatment as they present with advanced disease. However, there is an increasing incidence of urothelial cancer in Africa over the years due to urbanization. It is best that major investment is made in uro-oncological care to address the growing challenge of these subtypes.
\end{abstract}

Keywords: Non-muscle invasive bladder cancer; Radical cystectomy; Transurethral resection of bladder tumor

\section{Introduction}

Bladder cancer is the fourth most common cancer in male af-

Manuscript submitted May 29, 2019, accepted June 21, 2019

aDepartment of Urology and Andrology, Hopital General de Grand Yoff, Dakar, Senegal

bDepartment of Surgery, Liberia College of Physicians and Surgeons, Monrovia, Liberia

${ }^{c}$ UFR Sante, Universite de Thies, Senegal

${ }^{\mathrm{d} C}$ Corresponding Author: Ayun Cassell, Department of Urology and Andrology, Hopital General de Grand Yoff, Dakar, Senegal.

Email: ayuncasselliii@gmail.com

doi: https://doi.org/10.14740/wjon1210 ter prostate, lung and colorectal cancers, accounting for $6.6 \%$ of all cancer cases [1,2], and the 11th most common cancer in woman [1]. The global standardized incidence rate is 10.1 per 100,000 for men and 2.5 per 100,000 for women [2]. According to the Canadian Cancer Society, bladder cancer is the fifth most common cancer overall, accounting for 7,800 cases/ year [3]. Among the 81,190 estimated newly diagnosed $\mathrm{BC}$ in the US in 2018, nearly $75 \%$ were non-muscle invasive bladder cancer (NMIBC) [4]. A systemic review and meta-analysis by Adeloye et al in 2019, showed the pooled incidence of bladder cancer in Africa was 7.0 (95\% confidence interval: 5.8 - 8.3) per 100,000 population in men and 1.8 (95\% confidence interval: 1.2 - 2.6) per 100,000 in women [5]. The risks of bladder cancer appear to vary across world regions, correlating with smoking and occupational exposures to carcinogens in developed countries, and with chronic bladder urothelial irritation from Schistosoma hematobium infection in Africa and the Middle East [5].

Several studies and epidemiological profile in the developed nations have shown that approximately $70-75 \%$ of bladder cancers are NMIBC $[1,3,4,6]$. A few studies from Nigeria and Ethiopia (Forae et al [7], Biluts et al [8]) provided considerable rates of NMIBC as compared to western settings $70 \%$ and $85 \%$ respectively. NMIBC is defined as a superficial neoplasia confined to the mucosa, (including Ta which is a noninvasive papillary carcinoma and carcinoma in situ (CIS), which is flat and non-papillary) or lamina propria (T1) based on the American Joint Committee on Cancer (AJCC) staging system, also known as the tumor node metastases (TNM) classification [4]. Ta accounts for most NMIBC (60\%), whereas T1 and Tis (CIS) account for $30 \%$ and $10 \%$, respectively [3].

Patients with NMIBC should be placed into one of three risk categories (low, intermediate and high risk) for recurrence and progression based on their stage, grade, number and size of tumors, and whether CIS is present [9]. This allows the most appropriate management to be selected based on the patient's risk category [9].

Bladder cancer is a heterogeneous disease with a variable natural history [6]. In developed and industrialized nations, urothelial cancer is the most common histological type $[3,10]$. In much of Africa, the histological type has remained squamous cell carcinoma (SCC) due to the endemicity of schistosomiasis [11-13]. A review by Bowa et al revealed that cancer of the bladder in most of Africa is still predominantly SCC 
(53-69\%). However, there has been a notable increase in transitional cell carcinoma (TCC) in Africa (9-41\%) [11]. A 10year retrospective study of Salem et al in Egypt showed that there is a significant change of histological types from SCC to urothelial cancer; the incidence of associated bilharziasis decreased from $80 \%$ to $50 \%$; and a significant increase occurred in TCC from $20 \%$ to $66 \%$, with a significant decrease in SCC from $73 \%$ to $25 \%$ [14].

The TNM histological-based staging, which focuses on the luminal spread of the cancer across the bladder wall better applies to urothelial cancer, while with SCC, an organ-based staging is more suitable as most of the cancers associated with schistosomiasis had invaded the muscularis propria of the urinary bladder at the time of diagnosis $(\mathrm{P}<0.001)$ as reported by Rambau et al in Tanzania [15]. The management of TCC is mostly by transurethral resection of bladder tumor (TURBT) and adjuvant bladder instillation therapy [11]. Practically, SCC responds poorly to chemotherapy or radiotherapy and the main management of SCC is open surgery [11].

There is currently insufficient data regarding the standard of care of NMIBC in Africa. However, this review tends to highlight the challenges on the current management of NMIBC in Africa and compare outcomes to the current available guidelines in the western settings.

\section{Literature Search Methodology}

A literature review was conducted from 2000 to 2019 using Medline, African Journals Online, Google Scholar and PubMed. The English literature was searched using the term "non-muscle invasive bladder cancer" with tags (Africa/sub-Saharan Africa/ Nigeria/Ghana/Senegal/Liberia/Tanzania/Togo/Egypt/Benin/ Mali). A total of 47 articles were selected including retrospective studies, prospective studies, randomized trials, review articles, systemic review and meta-analysis and clinical guidelines.

Both abstracts and full text were reviewed for epidemiology, risk factors, clinical presentation (Table 1, [7, 8, 12, 13, $15,16-23])$, cystoscopic diagnosis, urine markers and cytology as well as TURBT, histological types (Table 2, [7, 8, 12, $13,15,16-23]$ ), intravesical adjuvant therapy and follow-up. A qualitative evaluation was performed on the various above parameters and a comparative analysis was made between the available African literature with reference to the current guidelines on bladder cancer by the European Urological Association (EAU), American Urological Association (AUA), Society of Urological Oncology (SUO), and the Canadian Urological Association.

\section{Literature Search Results}

Table $1[7,8,12,13,15,16-23]$ displays the demographics, age range, sex ratio and the commonest presenting symptoms of bladder cancer in most parts of sub-Saharan Africa.

Table $2[7,8,12,13,15,16-23]$ shows the mode of diagnosis mostly by white light cystoscopy, stage at presentation and histological types.

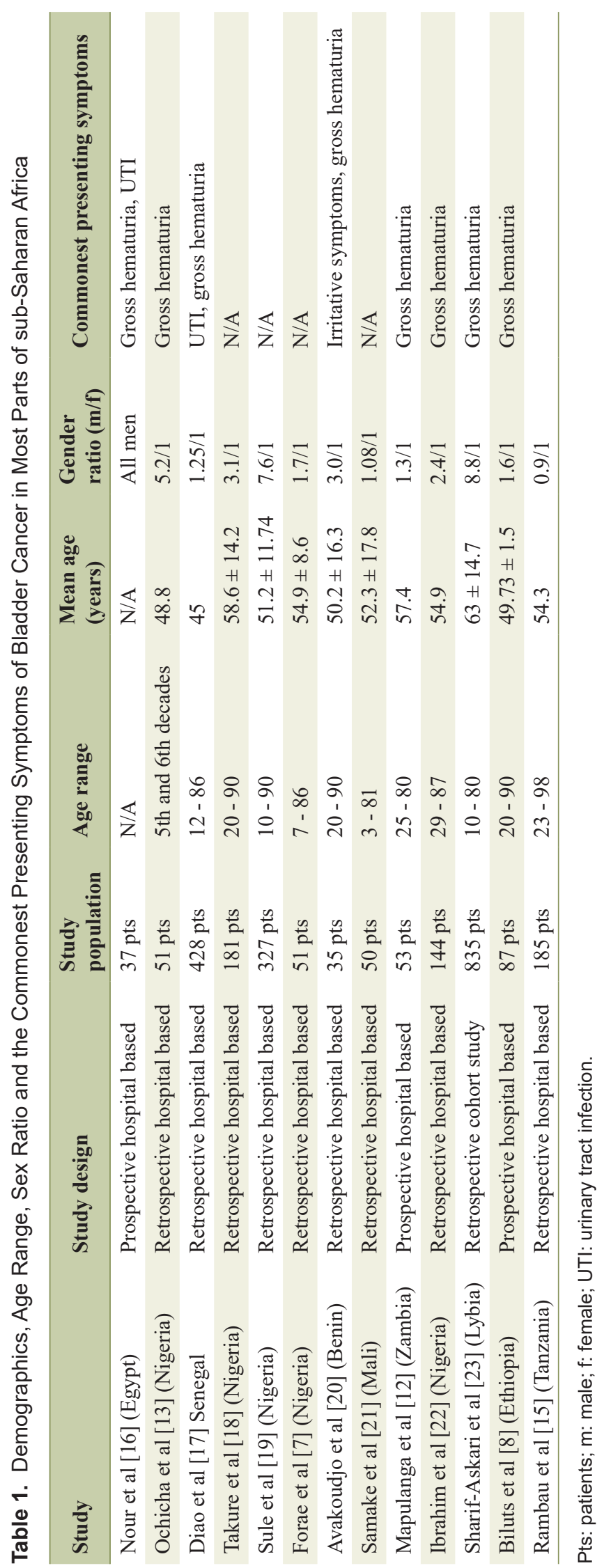


Table 2. Mode of Diagnosis (Mostly by White Light Cystoscopy), Stage at Presentation and Histological Types

\begin{tabular}{|c|c|c|c|c|c|}
\hline \multirow{2}{*}{ Study } & \multirow{2}{*}{ Mode of diagnosis } & \multirow{2}{*}{ Tumor stage at presentation } & \multicolumn{3}{|c|}{ Histopathological type of bladder cancer } \\
\hline & & & Urothelial cancer & Squamous cell & Others \\
\hline Nour et al [16] & Cystoscopy & NMIBC & & & \\
\hline Diao et al[17] & Cystoscopy & N/A & $\mathrm{N} / \mathrm{A}$ & $50.7 \%$ & $29.2 \%$ Schistosoma ova \\
\hline Takure et al [18] & N/A & N/A & $68.5 \%(122 / 181)$ & $19.9 \%$ & $11 \%$ Adenocarcinoma \\
\hline Avakoudjo et al [20] & Cystoscopy & N/A & N/A & 1 patient & 3 Adenocarcinomas \\
\hline Samake et al [21] & Cystoscopy & N/A & $20 \%$ & $76 \%$ & $4 \%$ \\
\hline Mapulanga et al [12] & & N/A & $30.2 \%$ & $60.4 \%$ & $9.4 \%$ Adenocarcinoma \\
\hline Ibrahim et al [22] & Cystoscopy & N/A & $30.5 \%$ & $63.8 \%$ & $5.7 \%$ \\
\hline
\end{tabular}

NMIBC: non-muscle invasive bladder cancer.

\section{Discussion}

\section{Epidemiology/risk factors}

The etiology of bladder cancer has been elucidated in most western literature mostly regarding urothelial cancer. Several studies $[2,3,5,10,11]$ have reported that smoking is a prominent risk factor for bladder cancer and the risk of recurrence is increased when smoking is continued [8]. Reports have shown that high-risk groups include workers in the following industries: printing, iron and aluminum processing, industrial painting, gas and tar manufacturing [2, 10]. According to Kassouf et al (2015) as stipulated in the Canadian Urological Association Guideline on the management of NMIBC, chronic inflammatory changes in the bladder (due to persistent bladder stones, recurrent urinary tract infections, chronic indwelling catheters and chemotherapeutic exposure are all relevant risk factors for bladder cancer [3].

Most of the African literatures on NMIBC are retrospective hospital-based studies and lack structured guideline available for bladder cancer [7-13]. The study populations as shown in Table 1 [7, 8, 12, 13, 15, 16-23] are heterogenous and conclusions cannot be simply drawn from a pool analysis of reported data. There are remarkable variations in the age range (Table 1, [7, 8, 12, 13, 15, 16-23]) as studies from Mali, Nigeria and Libya $[7,19,23]$ revealed patients with bladder tumor as young as 10 years and below. These tumors were however more of a variant histology or may have been congenital in origin. With the increase in life expectancy in some sub-Saharan regions, data from Nigeria, Ethiopia and Tanzania [8, 15, 19] showed a higher age range of diagnosis of bladder cancer at 90 years (Table 1, [7, 8, 12, 13, 15, 16-23]). Nevertheless, the median age in most studies was shown to be in the fifth decade (Table 1, [7, 8, 12, 13, 15, 16-23]). More males are found to be smokers than their female counterparts as well as earners for their family within agricultural and petrol industries in Africa. This put men at a higher risk for bladder cancer as revealed consistently in most studies.

Studies from Nigeria, Kenya, South Africa and Zambia $[11-13,24]$ have revealed that schistosoma endemicity has been a major etiological factor for bladder cancer. Retrospective reviews from Mali, Zambia, Tanzania, Nigeria and parts of North Africa displayed that $10-45 \%$ of patients diagnosed of SCC were found to have an associated bilharzial cystitis [11, $12,15,21]$. The histological type in these regions is more SCC than urothelial cancer, due to the relatively lower exposure to industrialized petrochemical and aromatic hydrocarbons in rural settings [5, 13, 18, 25].

Nevertheless, epidemiological studies in Egypt and other African data have shown that this historical trend is now taking a paradigm shift as the incidence of urothelial cancer is rising in Africa due to the urbanization and industrialization $[5,18$, 25]. A retrospective study of 192 patients by (Darre et al) in Togo found a high prevalence of urinary schistosomiasis with $28.1 \%$ tumor detection rate. Despite the findings urothelial was still the dominant histological type [26]. Data from Egypt, Libya, Ethiopia and some part of Nigeria are now showing a much higher incidence of urothelial cancer as compare to SCC (Table 2, [7, 8, 12, 13, 15, 16-23]). According to (Sharif-Askari et al) the incidence of this disease is expected to grow in developing countries because of increase in smoking popularity, the shift to sedentary life, diabetes mellitus, and obesity [23].

\section{Clinical presentation}

Comprehensive history, physical exam, followed by cystourethroscopy is required for initial evaluation of suspected bladder cancer [8]. The most common presenting symptom of 


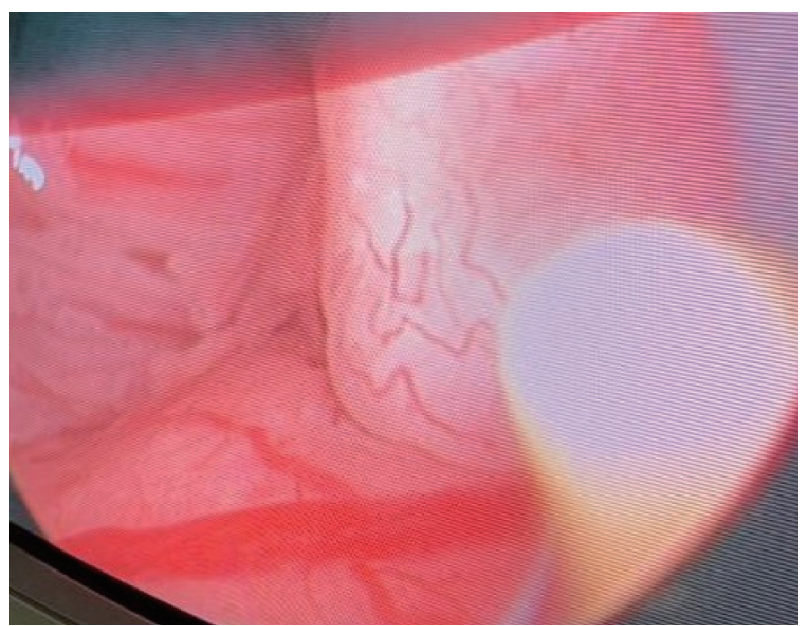

Figure 1. Evaluation of the bladder neck and prostatic urethral for mucosal changes using white light microscopy.

bladder cancer is painless macroscopic hematuria $[2,7,11,12]$ which occurs in about $85 \%$ of patients [2]. However, a review article on bladder cancer in sub-Saharan Africa by Bowa et al 2018 showed that urothelial cancer of the bladder presents with painless hematuria whereas SCC of the bladder is associated with painful hematuria, bladder mass and necroturia [11]. Retrospective analysis of most hospital-based studies in developing countries have shown that gross hematuria is the commonest presenting symptoms followed by irritative symptoms associated with UTI (Table 2, [7, 8, 12, 13, 15, 16-23]). A prospective study by (Edward et al) in UK involving 4,020 patients with hematuria showed macroscopic hematuria was associated with an $18.9 \%$ risk of urinary tract malignancy, while non-visible or microscopic hematuria was associated with a $4.8 \%$ risk of malignancy $[9,27]$.

\section{Diagnostic cystoscopy}

The diagnosis of bladder cancer ultimately depends on cystoscopic examination of the bladder $[1,3,9,10]$ and histological evaluation of the resected tissue [2] although about $4 \%$ of cases will be found incidentally, usually during imaging to investigate non-urological symptoms [9]. However, the detection rate for white light cystoscopy (WLC) can be limited (as low as $60 \%$ ) [1]. Strong evidence has shown that enhanced visualization methods using (hexyl aminolevulinate (HAL)-photodynamic diagnosis (PDD) or narrow band imaging (NBI)) may improve tumor detection and early recurrence $[1-3,10,28]$.

Blue light cystoscopy (BLC) or PDD requires preoperative intravesical instillation of hexaminolevulinate (HAL), a photosensitizer that preferentially accumulates in neoplastic cells [29]. Under blue light, red fluorescence is emitted by neoplastic cells. NBI improves visualization of tumor associated aberrant mucosal and submucosal vasculature, without the need for exogenous imaging agents [29].

A meta- analysis of data by Burger et al (2013) from 1,345 patients found that PDD or BLC detected significantly more Ta tumors $(14.7 \% ; \mathrm{P}<0.001)$ and CIS lesions $(40.8 \% ; \mathrm{P}<0.001)$

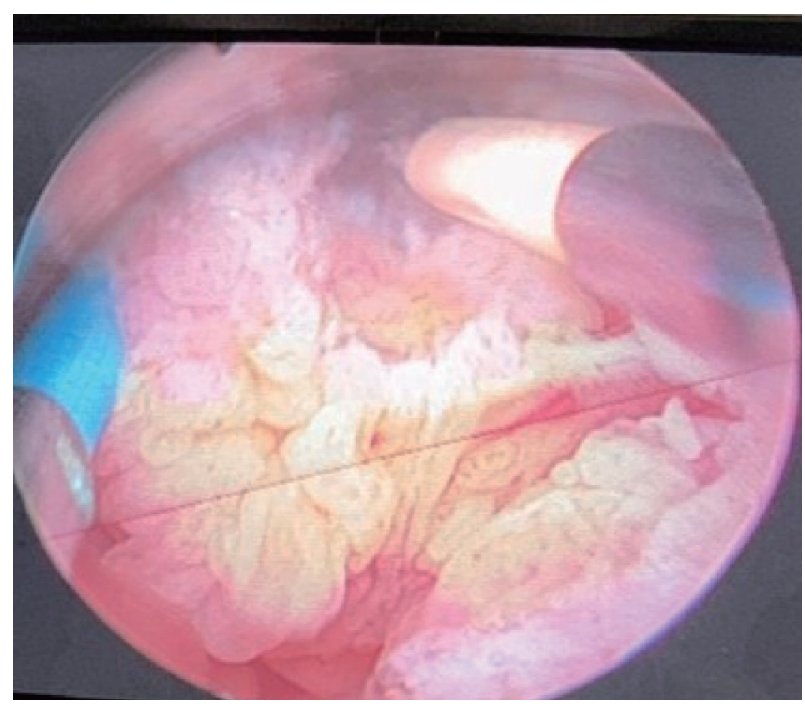

Figure 2. Transurethral resection of an exophytic bladder tumor in fraction using a bipolar resectoscope at the Hospital General de Grand Yoff, Dakar, Senegal.

than WLC $[1,30]$. A network meta-analysis by Lee et al (2015) found a lesser recurrence rate in patients undergoing NBI than patients undergoing WLC (OR, 0.48; 95\% CI, 0.26 - 0.95) but no significant difference in the recurrence rates of BLC and NBI treated patients $[1,31]$.

Both AUA and NICE recommend enhanced cystoscopy be offered at the time of TUR. EAU recommends PDD-guided biopsies in the setting of positive cytology but negative cystoscopy [10].

In the developing countries, the diagnosis of bladder cancer is still highly reliant on WLC and histology from TURBT which the standard of care is still shown in Figures 1, 2. Nevertheless, the absence of enhanced cystoscopy in these settings may affect the rate of recurrence after TURBT with poverty and lack of specialist facilities as confounding factors. Subsequently, more studies are needed to validate this effect as most of the histology is SCC that may require a radical cystectomy.

\section{Urinary markers and cytology}

Urine cytology has a high sensitivity for the detection of high-grade tumors but its sensitivity decreases (ranging from $4-31 \%$ ) for low-grade tumors $[1,2,10,28]$. In the case of positive cytology, the presence of urothelial cancer is highly probable, even if it cannot be visualized, and all necessary measures should be considered and performed (e.g., random biopsy, prostatic urethra biopsy and fluorescence cystoscopy) [28].

The AUA makes no recommendation regarding urine cytology in the primary assessment but advocates for its use in the surveillance of intermediate- and high-risk patients [28]. Conversely, EAU and NCCN guidelines recommend the use of urine cytology as an adjunct to cystoscopy at primary assessment and in the surveillance of high-risk tumors [10].

Although several urine-based tumor markers have been investigated and developed (e.g., NMP22, BTA test, immuno- 
Cyt, microsatellite analysis, CYFRA21-1, fluorescence in situ hybridization (FISH), and Lewis-X), their low sensitivity and low specificity have prevented their application to NMIBC diagnosis and prognosis [1]. Nevertheless, a study by Soria et al (2018) reported that FISH has been shown to be useful in specific scenarios, mostly as a reflex test and in the setting of equivocal urinary cytology [32]. In a patient with NMIBC, a clinician may use biomarkers to assess response to intravesical bacillus Calmette-Guerin (BCG) (UroVysion ${ }^{\circledR}$ FISH) and adjudicate equivocal cytology [33].

\section{Management}

\section{TURBT}

TURBT under regional or general anesthesia remains the gold standard for the management of NMIBC. The histological type and grade of the tumor, as well as the presence, depth, and type of the tumor invasion should be determined [1-4, 6, 9, 28, 33]. Bimanual examination of the bladder should be performed under anesthesia before prepping and draping unless the tumor is clearly small and noninvasive and should be repeated after the resection. Fixation or persistence of a palpable mass after resection suggests locally advanced disease [2].

The EAU offers specifics about TUR technique, including en bloc resection of small papillary tumors including the underlying bladder wall, while larger tumors should be resected in fractions and include the underlying detrusor muscle, and edges of the resection area [10]. Despite some bleeding, cauterization must be avoided as much as possible during the resection. Excessive cautery artefact may make interpretation of tissue by pathologist difficult [28].

In agreement with published data, EAU guidelines strongly recommend re-TUR in patients with high-grade and T1 tumors and always when the pathologist has reported that the specimen contained no muscle tissue. Most authors perform the procedure between 2 and 6 weeks after initial TURB [1-4, $6,9,28,33,34]$. Because of the frequent detection of persistent disease at the location of the original tumor, the deep resection of this site is advocated [15].

Repeating TUR in select cases is associated with discovering persistent tumor $[35,36]$, more accurate staging (especially if no detrusor muscle in the initial TUR) [34], improved recurrence free survival and response to BCG [10].

The management of bladder cancer in sub-Saharan Africa is unclear. The recommended guidelines require great investment which is minimal to absent in most settings in Africa [17, $18,20,37]$. The very few specialists must manage patients who will apparently become loss to follow-up and present later with an advanced stage of the disease. Very few studies have reported on TURBT including studies from Egypt, Nigeria and Ethiopia [8, 16, 21, 22]. Even open surgery (partial cystectomy, radical cystectomy and urinary diversion) may become a nightmare due to the lack of neoadjuvant and adjuvant chemotherapy. The outcome of cystectomy and diversion have been reported with some adverse outcome including metabolic acidosis, sepsis and multiple organ dysfunction (Biluts et al,
Ibrahim et al) $[8,22]$. A systemic review and meta-analysis of 22 articles on the incidence of bladder cancer in Africa by (Adeloye et al) displayed ureterosigmoidostomy and biopsy were the comment procedures in $27.7 \%$ of case as $20.8 \%$ of patients presented with advanced disease in Africa which is remarkably higher than data in the developed nations [5].

\section{Prostate urethral biopsy}

The involvement of urothelial carcinoma at the prostatic urethra (PU) is a negative prognostic factor for recurrence and progression [10]. Although the exact risk of prostatic urethra or duct involvement is not known, it seems to be higher if the tumor is located on the trigone or bladder neck, in the presence of bladder CIS and multiple tumors $[2,38]$. The biopsy is taken from abnormal areas and from the precollicular area (between the 5 and 7 o'clock position) using a resection loop [2]. Both the EAU and AUA recommend prostate urethral biopsy for patients with a history of NMIBC, negative cystoscopy but positive urine cytology [10].

\section{Adjuvant intravesical chemotherapy}

The choice of therapy may be considered on an individual basis according to what risk is acceptable for the patient and the urologist [2]. A meta-analysis of seven trials by Sylvester et al [39] confirmed that one immediate intravesical instillation of chemotherapy significantly decreased the risk of recurrence after TURBT, not only in patients with low-risk NMIBC but also in patients with stage Ta-T1 single and multiple bladder cancers [6]. In the most recent systematic review, it was shown that a single instillation (SI) reduced the 5-year recurrence rate by $14 \%$ [1]. In EAU guidelines, it was reported that mitomycin $\mathrm{C}$ (MMC), epirubicin, and pirarubicin have all shown a beneficial effect $[1,2]$. Numerous trials evaluated the use of MMC, with 20 - $60 \mathrm{mg}$ MMC administered once a week for 4 - 8 weeks, with or without maintenance once every 2 weeks to 3 months for 6 months to 2 years [6]. However, adjuvant intravesical therapy should be omitted in patients with hematuria, with overt or possible bladder perforation and after extensive resection $[1-3,28]$.

\section{1) Low risk}

Low-risk tumors, classified as single TaG1 tumors, $3 \mathrm{~cm}$ in diameter, have a recurrence probability of $15-24 \%$ and $31-46 \%$ at 1 year and 5 years, respectively [4]. For patients with these low-risk tumors, one immediate instillation is considered adequate.

\section{2) Intermediate risk}

This risk group contains all other tumors between the low- and high-risk groups, namely Ta-1, grade 1-2, multifocal, and $>3$ 
$\mathrm{cm}$ in diameter [6]. Based on the European Organization for Research and Treatment of Cancer (EORTC) risk assessment, the calculated probabilities of recurrence at 1 year and 5 year, respectively, are $24-38 \%$ and $46-62 \%$ [6]. The AUA/SUO guideline [33] recommends that intermediate-risk patients are considered for administration of a 6-week course of intravesi$\mathrm{cal}$ induction chemotherapy followed by maintenance therapy if there is a complete clinical response [33].

\section{3) High risk}

In a high-risk patient with newly diagnosed CIS, high-grade $\mathrm{T} 1$, or high-risk Ta urothelial carcinoma, a clinician should administer a 6-week induction course of BCG [33].

\section{Advances in intravesical immunotherapy (BCG)}

BCG is indicated not only in high-risk disease, but also in intermediate-risk disease because the benefit of BCG on recurrence and progression is greatest in both groups [1]. The protocol of induction BCG should consist of six weekly intravesical instillations, followed by maintenance consisting of three weekly treatments at 3 month, and 6 months, for a total of 36 months [1]. Treatments usually begin 2 - 4 weeks after the TURBT, allowing time for the re-epitheliazation to minimize the potential for intravasation of live bacteria [2]. Four meta-analyses (Shelley et al 2001; Han et al 2006, Shelley et al 2004, Bohle et al 2003) have confirmed that BCG after TUR is superior to TUR alone or TUR and chemotherapy for the prevention of recurrence of NMIBC in patients with Ta and T1 tumors [40-43].

The main action of BCG in the bladder is to mediate the production of adhesion and costimulatory molecules after its internalization into the tumor cells, thus increasing the binding capacity of $\mathrm{T}$ lymphocytes and neutrophils to receptors, leading to an enhanced activity against tumor cells resulting in their apoptosis and inhibition of their replication [44].

Relatively little has been published about the efficacy of current therapies in treating NMIBC in elderly patients. A multicenter trial found that overall response to either $\mathrm{BCG}$ or $\mathrm{BCG}$ plus interferon was decreased in participants $\geq 80$ years of age compared with younger patients [45]. BCG unresponsive disease should be defined as: 1) patients with recurrent high grade T1 disease within 6 months of their primary tumor after at least one course of BCG or patients who have failed at least two courses of BCG with either 2) persistent or recurrent pure papillary (Ta) disease within 6 months or 3) persistent or recurrent CIS within 12 months [46, 47].

A prospective study of 37 patients by Nour et al in Egypt who underwent TURBT were evaluated for low-dose BCG efficacy (recurrence with or without progression) and safety by documentation of minor and/or major side effects. There were no major or severe side effects and no treatment discontinuations. Besides North Africa, the use of BCG in the sub-Saharan nations as an adjuvant intravesical therapy is sparse or underreported [16].

\section{Treatment of failure following intravesical therapy}

In patients with BCG-refractory high-risk NMIBC, radical cystectomy is recommended $[2,3,6,10]$. In patients with BCG relapse, BCG plus interferon, gemcitabine, or re-induction with BCG are valid options when patients are not suitable for or refuse radical cystectomy $[3,10]$.

\section{The role of early cystectomy}

Although the initial response rate to BCG therapy in patients with CIS can be above $80 \%$, patients whose condition fails to respond have a $50 \%$ chance of disease progression and a higher likelihood of disease-specific mortality [2]. Therefore, some experts consider it reasonable to propose immediate cystectomy to patients with NMIBC who are at high risk of progression [2].

Early radical cystectomy may be advised for very highrisk patients: T1 high grade with variant features; T1 high grade with lymphovascular invasion, multiple and/or large T1 high grade; T1 high grade with concomitant bladder/prostatic CIS; persistent T1 high grade on restaging TUR; early highgrade recurrence at 3 months; and invasive tumors involving bladder diverticula $[3,10]$.

A review article by Bowa et al (2018) reported that the major histological type in Africa is SCC and these tumors are locally invasive spreading from the fundus to the bladder neck rather than the transmural spread seen in urothelial bladder cancer [11]. Therefore, it is efficacious that these tumors are best treated with radical cystectomy than the conventional TURBT for non-muscle invasive urothelial bladder tumor; as the patients are relatively young and healthy, and there is no risk of urethral recurrence, unlike with urothelial cancer [11, 24, 47]. Heyns et al also stated that unfortunately, many patients in Africa still present with advanced and inoperable bladder cancer, and many do not have access to healthcare facilities that can provide a cure and a good quality of life by means of radical cystectomy and neobladder construction [24].

\section{Follow-up}

Cystoscopy at 3 months following TURBT is recommended for all patients. Generally, cystoscopy with urine cytology (or other urine marker) is recommended every 3 to 4 months for 2 years, then every 6 months for years 3 and 4 , then yearly thereafter [3]. Patients with low-risk Ta tumors may undergo cystoscopy at 3 and 12 months, then annually (level of evidence 3 ). Upper tract imaging every 1 to 2 years is recommended for patients with high-risk NMIBC [3].

Patient follow-up is a challenge in sub-Saharan Africa due to low socioeconomic status, poor accessibility to health care, lack of adequate or accessible specialist care as well as poor insight of their disease condition. Based on the aforementioned, even patients diagnosed of low-risk NMIBC can return with a more advanced stage of the disease. Studies from Egypt, Nigeria and Ethiopia [8, 16, 22] have recommended patient follow-up after treatment from 3 months up to 4 years using 
cystoscopy with bladder wash cytology [16].

\section{Conclusions}

Critical steps in the management of NMIBC are to prevent tumor recurrence which includes TURBT for staging and histological diagnosis. A second TURBT for high grade tumor, T1 tumors and intravesical adjuvant chemotherapy and immunotherapy are essential to reduce recurrence rate. Nevertheless, variant histology, multiple, progressive and recurrent high-grade tumors are best treated with early radical cystectomy. The African literature is scanty on the management of NMIBC. Most of the histological types are squamous cell bladder cancer and may not conform to transurethral resection only but rather radical cystectomy. Most of these patients are not suitable for any form of treatment as they present with advanced disease. However, there is an increasing incidence of urothelial cancer in Africa over the years due to urbanization. It is best that major investment is made in uro-oncological care to address the growing challenge of these subtypes.

\section{Acknowledgments}

Special thanks to the Department of Urology and Andrology of the Hospital General de Grand Yoff.

\section{Conflict of Interest}

None.

\section{Financial Disclosure}

No external funding available to disclose.

\section{Author Contributions}

Conceptual design: AC, BY, MJ. Analysis, drafting and critical revision of the article: $\mathrm{AC}, \mathrm{BY}, \mathrm{YD}, \mathrm{AD}, \mathrm{MM}$. Final approval of the article: LN, IL, SG. Critical review and analysis: MN.

\section{Abbreviations}

NMIBC: non-muscle invasive bladder cancer; RC: radical cystectomy; TURBT: transurethral resection of bladder tumor; AUA: American Urological Association; EAU: European Association of Urology; SCC: squamous cell carcinoma; SUO: Society of Urologic Oncology; UC: urothelial cancer

\section{References}

1. Kamat AM, Bagcioglu M, Huri E. What is new in non- muscle-invasive bladder cancer in 2016? Turk J Urol. 2017;43(1):9-13.

2. Anastasiadis A, de Reijke TM. Best practice in the treatment of nonmuscle invasive bladder cancer. Ther Adv Urol. 2012;4(1):13-32.

3. Kassouf W, Traboulsi SL, Kulkarni GS, Breau RH, Zlotta A, Fairey A, So A, et al. CUA guidelines on the management of non-muscle invasive bladder cancer. Can Urol Assoc J. 2015;9(9-10):E690-704.

4. Shen PL, Lin ME, Hong YK, He XJ. Bladder preservation approach versus radical cystectomy for high-grade non-muscle-invasive bladder cancer: a meta-analysis of cohort studies. World J Surg Oncol. 2018;16(1):197.

5. Adeloye D, Harhay MO, Ayepola OO, Dos Santos JP, David RA, Ogunlana OO, Gadanya M, et al. Estimate of the incidence of bladder cancer in Africa: A systematic review and Bayesian meta-analysis. Int J Urol. 2019;26(1):102112.

6. Hendricksen K, Alfred Witjes JA. Treatment of intermediate-risk Non-Muscle-Invasive Bladder Cancer (NMIBC). European Urology Supplements. 2007;6(14):800-808.

7. Forae GD, Ugiagbe EE, Mekoma DF. A descriptive study of bladder tumors in Benin City, Nigeria: An analysis of histopathological patterns. Saudi Surg J. 2016;4:113-117.

8. Biluts H, Minas E. Bladder tumours at Tikur Anbessa Hospital in Ethiopia. East and Central African Journal of Surgery. 2011;16(1):57-64.

9. Mostafid H, Bryan RT, Rees J. Diagnosis and treatment of 13 non-muscle-invasive bladder cancer. Trends in Urology \& Men's Health. 2015;6(2):23-27.

10. Woldu SL, Bagrodia A, Lotan Y. Guideline of guidelines: non-muscle-invasive bladder cancer. BJU Int. 2017;119(3):371-380.

11. Bowa K, Mulele C, Kachimba J, Manda E, Mapulanga V, Mukosai S. A review of bladder cancer in Sub-Saharan Africa: A different disease, with a distinct presentation, assessment, and treatment. Ann Afr Med. 2018;17(3):99105.

12. Mapulanga V, Labib M, Bowa K. Pattern of bladder cancer at University Teaching Hospital, Lusaka, Zambia in the era of HIV Epidemic. Med J Zambia. 2012;39(1):2226.

13. Ochicha O, Alhassan S, Mohammed AZ, Edino ST, Nwokedi EE. Bladder cancer in Kano-a histopathological review. West Afr J Med. 2003;22(3):202-204.

14. Salem HK, Mahfouz S. Changing patterns (age, incidence, and pathologic types) of schistosoma-associated bladder cancer in Egypt in the past decade. Urology. 2012;79(2):379-383.

15. Rambau PF, Chalya PL, Jackson K. Schistosomiasis and urinary bladder cancer in North Western Tanzania: a retrospective review of 185 patients. Infect Agent Cancer. 2013;8(1):19.

16. Nour HH, Gobashy SE, Kamal AM, Elbaz AG, Roshdy MA, Kamel AI. Low-dose bacille Calmette-Guerin for non-muscle-invasive bladder cancer: Results of a prospective study. Arab J Urol. 2015;13(4):238-243.

17. Diao B, Amath T, Fall B, Fall PA, Dieme MJ, Steevy NN, 
Ndoye AK, et al. [Bladder cancers in Senegal: epidemiological, clinical and histological features]. Prog Urol. 2008;18(7):445-448.

18. Takure AO, Odubanjo MO, Adebayo SA, Oluwasola O, Shittu OB, Okeke LI, Fadimu OA, et al. Histopathologic pattern of bladder cancers in Ibadan Southwest Nigeria: an update. J West Afr Coll Surg. 2015;5(2):17-42.

19. Sule A A, Ochicha O, Ibrahim Y, Adam S, Abubakar A, Haruna M S. Update on bladder cancer in Kano, Northern Nigeria. Niger J Basic Clin Sci. 2017;14:26-29.

20. Avakoudjo JDG, Hounnasso PP, Gouissi SAG. Bladder tumor: epidemiologic and diagnostic aspect at University Hospital of Cotonou. Journal De La Societe De Biologie Clinique Du Benin. 2015;022:77-80

21. Samake L. Aspects epidemiologiques et histopathologiques des cancers de la vessie au Mali. 2014. https://scholar.google.com/scholar

22. Ibrahim AG, Aliyu S. Bladder cancer a ten-year experience In Maiduguri North Eastern Nigeria. IJSER. 2015;6(2):1551-1560.

23. Saheb Sharif-Askari N, Bendardaf R, Saheb Sharif-Askari F, El Tabbal AM, El Ayan MA. Incidence of bladder cancer in Benghazi, Libya over the past three decades. Sci Rep. 2018;8(1):10822.

24. Heyns CF, van der Merwe A. Bladder cancer in Africa. Can J Urol. 2008;15(1):3899-3908.

25. Felix AS, Soliman AS, Khaled H, Zaghloul MS, Banerjee M, El-Baradie M, El-Kalawy M, et al. The changing patterns of bladder cancer in Egypt over the past 26 years. Cancer Causes Control. 2008;19(4):421-429.

26. Darre T, Kpatcha M, Tchaou M, Amegbor K, Sonhaye L, N'Timon B, Napo-Koura G. [Histological aspect of urinary schistosomiasis in Togo: results of a cohort of 192 cases]. Bull Soc Pathol Exot. 2015;108(2):124-125.

27. Edwards TJ, Dickinson AJ, Natale S, Gosling J, McGrath JS. A prospective analysis of the diagnostic yield resulting from the attendance of 4020 patients at a protocoldriven haematuria clinic. BJU Int. 2006;97(2):301-305; discussion 305.

28. Marko Babjuk. Transurethral resection of non-muscleinvasive bladder cancer. European Urology Supplements. 2009;8(7):542-548.

29. Chang TC, Marcq G, Kiss B, Trivedi DR, Mach KE, Liao JC. Image-guided transurethral resection of bladder tumors - current practice and future outlooks. Bladder Cancer. 2017;3(3):149-159.

30. Burger M, Grossman HB, Droller M, Schmidbauer J, Hermann G, Dragoescu O, Ray E, et al. Photodynamic diagnosis of non-muscle-invasive bladder cancer with hexaminolevulinate cystoscopy: a meta-analysis of detection and recurrence based on raw data. Eur Urol. 2013;64(5):846-854.

31. Lee JY, Cho KS, Kang DH, Jung HD, Kwon JK, Oh CK, Ham WS, et al. A network meta-analysis of therapeutic outcomes after new image technology-assisted transurethral resection for non-muscle invasive bladder cancer: 5-aminolaevulinic acid fluorescence vs hexylaminolevulinate fluorescence vs narrow band imaging. BMC Cancer. 2015;15:566.
32. Soria F, Droller MJ, Lotan Y, Gontero P, D'Andrea D, Gust KM, Roupret M, et al. An up-to-date catalog of avfailable urinary biomarkers for the surveillance of non-muscle invasive bladder cancer. World J Urol. 2018;36(12):19811995.

33. Chang SS, Boorjian SA, Chou R, Clark PE, Daneshmand S, Konety BR, Pruthi R, et al. Diagnosis and treatment of non-muscle invasive bladder cancer: AUA/SUO guideline. J Urol. 2016;196(4):1021-1029.

34. Herr HW, Donat SM. Quality control in transurethral resection of bladder tumours. BJU Int. 2008;102(9 Pt B):1242-1246.

35. Mariappan P, Zachou A, Grigor KM, Edinburgh Uro-Oncology G. Detrusor muscle in the first, apparently complete transurethral resection of bladder tumour specimen is a surrogate marker of resection quality, predicts risk of early recurrence, and is dependent on operator experience. Eur Urol. 2010;57(5):843-849.

36. Brausi M, Collette L, Kurth K, van der Meijden AP, Oosterlinck W, Witjes JA, Newling D, et al. Variability in the recurrence rate at first follow-up cystoscopy after TUR in stage Ta T1 transitional cell carcinoma of the bladder: a combined analysis of seven EORTC studies. Eur Urol. 2002;41(5):523-531.

37. Abdulkadir A, Alhaji SA, Sanusi HM. Pattern of urological cancers in Kano: North-western Nigeria. Sub-Saharan Afr J Med. 2016;3:182-187.

38. Mungan MU, Canda AE, Tuzel E, Yorukoglu K, Kirkali Z. Risk factors for mucosal prostatic urethral involvement in superficial transitional cell carcinoma of the bladder. Eur Urol. 2005;48(5):760-763.

39. Sylvester RJ, Oosterlinck W, van der Meijden AP. A single immediate postoperative instillation of chemotherapy decreases the risk of recurrence in patients with stage Ta T1 bladder cancer: a meta-analysis of published results of randomized clinical trials. J Urol. 2004;171(6 Pt 1):21862190, quiz 2435.

40. Shelley MD, Kynaston H, Court J, Wilt TJ, Coles B, Burgon $\mathrm{K}$, Mason MD. A systematic review of intravesical bacillus Calmette-Guerin plus transurethral resection vs transurethral resection alone in Ta and $\mathrm{T} 1$ bladder cancer. BJU Int. 2001;88(3):209-216.

41. Shelley MD, Wilt TJ, Court J, Coles B, Kynaston H, Mason MD. Intravesical bacillus Calmette-Guerin is superior to mitomycin $\mathrm{C}$ in reducing tumour recurrence in high-risk superficial bladder cancer: a meta-analysis of randomized trials. BJU Int. 2004;93(4):485-490.

42. Han RF, Pan JG. Can intravesical bacillus Calmette-Guerin reduce recurrence in patients with superficial bladder cancer? A meta-analysis of randomized trials. Urology. 2006;67(6):1216-1223.

43. Bohle A, Leyh H, Frei C, Kuhn M, Tschada R, Pottek T, Wagner W, et al. Single postoperative instillation of gemcitabine in patients with non-muscle-invasive transitional cell carcinoma of the bladder: a randomised, doubleblind, placebo-controlled phase III multicentre study. Eur Urol. 2009;56(3):495-503.

44. Verdeja-Robles CA, Turcio-Aceves O, Hernandez-Ibarra MA, Barragan-De la Cruz M, Sanchez-Pereda D, et al. 
Compared efficacy of intravesical BCG Vs Mitomycin-C, and other dual therapies in non-muscle invasive bladder cancer. Canc Therapy \& Oncol Int J. 2018;10(5):555798.

45. Erlich A, Zlotta AR. Treatment of bladder cancer in the elderly. Investig Clin Urol. 2016;57(Suppl 1):S26-35.

46. Steinberg RL, Thomas LJ, O'Donnell MA. Bacillus Cal-
mette-Guerin $(\mathrm{BCG})$ treatment failures in non-muscle invasive bladder cancer: what truly constitutes unresponsive disease. Bladder Cancer. 2015;1(2):105-116.

47. el-Mawla NG, el-Bolkainy MN, Khaled HM. Bladder cancer in Africa: update. Semin Oncol. 2001;28(2):174178. 